\title{
Ring Geometries, Two-Weight Codes, and Strongly Regular Graphs
}

\author{
Eimear Byrne, Marcus Greferath, and Thomas Honold
}

\begin{abstract}
It is known that a linear two-weight code $C$ over a finite field $\mathbb{F}_{q}$ corresponds both to a multiset in a projective space over $\mathbb{F}_{q}$ that meets every hyperplane in either $a$ or $b$ points for some integers $a<b$, and to a strongly regular graph whose vertices may be identified with the codewords of $C$.

Here we extend this classical result to the case of a ring-linear code with exactly two nonzero homogeneous weights and multisets of points in an associated projective ring geometry. We will show that a two-weight code over a finite Frobenius ring gives rise to a strongly regular graph, and we will give some constructions of two-weight codes using ring geometries. These examples all yield infinite families of strongly regular graphs with non-trivial parameters.
\end{abstract}

\section{Introduction}

It has been known since the 1970's that there are connections between certain sets in a projective geometry, partial difference sets, codes with two nonzero weights, and strongly regular graphs. The equivalence of classes of these objects was first observed by Delsarte [4, 5], and since then, many other constructions have been discovered (see [6] for a survey).

More recently, it has been observed that a linear code over $\mathbb{Z}_{4}$ with exactly two nonzero Lee weights also determines a strongly regular graph [2], so that a correspondence may be drawn between certain distance invariant non-linear binary codes and such graphs. In this paper, we show that a linear code over a finite Frobenius ring with two nonzero homogeneous weights also determines a strongly regular graph. In addition, we give constructions of two-weight codes over finite chain rings determined by point sets of associated projective geometries. These point sets provide new constructions of non-trivial strongly regular graphs. This indicates that projective geometries over rings may be used to determine new families of strongly regular graphs.

\section{Preliminaries: Finite Frobenius rings and homogeneous weights}

The merits of studying properties of linear codes over finite rings has been recognized since the end of the eighties (cf. [23, 14]). Two important foundational results by J. Wood [28, 29] state that the MacWilliams' equivalence and duality theorems can be extended for linear codes over a very large class of rings, namely the finite Frobenius rings. A recent paper by Wood [30] yields the 
final justification for the belief that the finite Frobenius rings are the appropriate class for algebraic coding theory over rings. The reader should note however, that the Frobenius property of the ring can be dropped by passing to a suitable module (a quasi-Frobenius module of the given ring) serving as alphabet for ring-linear coding theory (cf. [12]).

Detailed from the theory of quasi-Frobenius and Frobenius rings can be found in [9, 10, 18, Here, we briefly describe properties of such rings in the finite case.

For a finite ring $R$ denote by $\widehat{R}:=\operatorname{Hom}_{\mathbb{Z}}\left(R, \mathbb{C}^{\times}\right)$the character module of $R$. $\widehat{R}$ has a natural $R$-bimodule structure determined by $\chi^{r}(x):=\chi(r x)$ and ${ }^{r} \chi(x):=\chi(x r)$ for all $r, x \in R$ and each character $\chi \in \widehat{R}$.

A finite ring $R$ is called a Frobenius ring if it satisfies any of the following equivalent conditions:

(i) ${ }_{R}\left(\operatorname{Soc}\left({ }_{R} R\right)\right) \cong{ }_{R}(R / \operatorname{Rad}(R))$;

(ii) $\left(\operatorname{Soc}\left(R_{R}\right)\right)_{R} \cong(R / \operatorname{Rad}(R))_{R}$;

(iii) ${ }_{R} R \cong{ }_{R} \widehat{R}$;

(iv) $R_{R} \cong \widehat{R}_{R}$;

(v) $\operatorname{Soc}\left({ }_{R} R\right)$ is a principal left ideal;

(vi) $\operatorname{Soc}\left(R_{R}\right)$ is a principal right ideal.

Conditions (iii) and (iv) say that $R$ has a left (resp. right) generating character $\chi$, which means $\widehat{R}=\left\{{ }^{r} \chi \mid r \in R\right\}$ (resp. $\widehat{R}=\left\{\chi^{r} \mid r \in R\right\}$ ). It can be shown that a character is left generating if and only if it is right generating.

Homogeneous weights were first introduced by I. Constantinescu and W. Heise [3] in the context of linear codes over integer residue rings. Such a weight function has the property of taking a constant value on sets of associated ring elements.

Weights with these properties have been revisited and generalized in different ways (cf. [11, 13, 20, [15]). Here, we follow the line in [11, 13] which works without restrictions on the underlying finite ring.

Definition 2.1 Let $R$ be a finite ring and let $w: R \Longrightarrow \mathbb{R}$ be a map satisfying $w(0)=0$ and $w(x) \geq 0$ for all $x \in R$. The map $w$ is called a (left) homogeneous weight, if $w(0)=0$ and the following is true:

(H1) If $R x=R y$ then $w(x)=w(y)$ for all $x, y \in R$.

(H2) There exists a real number $\gamma$ such that

$$
\sum_{y \in R x} w(y)=\gamma|R x| \quad \text { for all } x \in R \backslash\{0\} .
$$

The number $\gamma$ may be thought of as the average value of the weight function, and the condition (H2) simply states that this average is the same on all nonzero principal left ideals. 
Example 2.2 We give some examples of finite rings with homogeneous weight functions. Observe that in 4 , the a non-zero ring element has been assigned weight zero.

(a) On every finite field $\mathbb{F}_{q}$ the Hamming weight is a homogeneous weight of average value $\gamma=\frac{q-1}{q}$

(b) On $\mathbb{Z}_{4}$ the Lee weight is homogeneous with $\gamma=1$.

(c) On a finite chain ring $R$ with residue field $\operatorname{GF}(q)$ the weight

$$
w: R \longrightarrow \mathbb{R}, \quad x \mapsto \begin{cases}0 & \text { if } x=0, \\ \frac{q}{q-1} & \text { if } x \in \operatorname{Soc}(R), x \neq 0, \\ 1 & \text { otherwise }\end{cases}
$$

is a left and right homogeneous weight with $\gamma=1$.

(d) On the ring $\mathbb{F}_{2} \oplus \mathbb{F}_{2}$ the weight $w$ defined by $w(0,0)=w(1,1)=0, w(1,0)=w(0,1)=2$ is a homogeneous weight with $\gamma=1$.

(e) On the (local) ring $R=\mathbb{F}_{2}[X, Y] /\left(X^{2}, Y^{2}, X Y\right)$ of order 8 the weight

$$
w: R \longrightarrow \mathbb{R}, \quad x \mapsto \begin{cases}0 & \text { if } x=0, \\ 2 & \text { if } x \in \operatorname{Rad}(R) \backslash\{0\}=\{X, Y, X+Y\}, \\ \frac{1}{2} & \text { otherwise, }\end{cases}
$$

is a homogeneous weight with $\gamma=1$.

As is common in coding theory, a weight $w$ on a finite ring $R$ will be extended to a weight on the $R$-module ${ }_{R} R^{n}$ in the obvious way:

$$
w: R^{n} \longrightarrow \mathbb{R}, \quad w\left(c_{1}, \ldots, c_{n}\right)=\sum_{i=1}^{n} w\left(c_{i}\right) .
$$

It can be shown that up to the choice of $\gamma$, every finite ring admits a unique (left) homogeneous weight (cf. [13, Thm. 1.3]). For the investigations here a further observation by Honold [21] is important and is used in the proof of Proposition 5.3.

Proposition 2.3 Let $R$ be a finite Frobenius ring with generating character $\chi$. Then the (left) homogeneous weight on $R$ has the form

$$
w: R \longrightarrow \mathbb{R}, \quad x \mapsto \gamma\left[1-\frac{1}{\left|R^{\times}\right|} \sum_{u \in R^{\times}} \chi(u x)\right]
$$

for some real number $\gamma$.

The following important fact, stated in [13, Lem. 1.5, Rem. 1.7(b)] and will be used for our computations later (see Lemma 4.1).

Remark 2.4 If $w$ is a (left) homogeneous weight of average value $\gamma \neq 0$ on the finite ring $R$, then $R$ is a Frobenius ring if and only if: 


$$
\sum_{y \in I} w(y)=\gamma|I| \quad \text { for all }\{0\} \neq I \leq{ }_{R} R
$$

Note, for example, that the ring of Ex. 2.2(e) is not a Frobenius ring, since its socle is not cyclic. Indeed,

$$
\sum_{x \in \operatorname{Rad}(R)} w(x)=6 \neq|\operatorname{Rad}(R)|
$$

for the given weight.

It has been shown in 20, that if $R$ is a finite Frobenius ring, then every left homogeneous weight is also right homogeneous, clearly with the same average value $\gamma$. This can also be inferred from Proposition 2.3 and the equation

$$
\sum_{u \in R^{\times}} \chi(u x)=\sum_{\substack{\psi \in \widehat{R} \\ \psi \text { generating }}} \psi(x)=\sum_{u \in R^{\times}} \chi(x u) .
$$

We shall call the unique homogeneous weight of average value $\gamma=1$ the normalized homogeneous weight and denote it by $\mathrm{w}_{\text {hom }}$.

Finally, the proof of Lemma 5.2 uses the notion of Möbius inversion for functions on finite poset:1 For details see [1, Ch. IV], [24], 25, Ch. 3.6], or [27].

Let $P$ be a finite poset. Consider the function $\mu: P \times P \longrightarrow \mathbb{R}$, defined recursively by the equations $\mu(x, x)=1$ and

$$
\mu(y, x)= \begin{cases}-\sum_{y<t \leq x} \mu(t, x) & \text { if } y<x, \\ 0 & \text { if } y \not \leq x .\end{cases}
$$

The map $\mu$ is called the Möbius function of $P$ and induces for arbitrary pairs of real-valued functions $f, g$ on $P$ the equivalence:

$$
g(x)=\sum_{y \leq x} f(y) \quad \text { for all } x \in P \quad \Longleftrightarrow \quad f(x)=\sum_{y \leq x} g(y) \mu(y, x) \quad \text { for all } x \in P .
$$

This equivalence is usually referred to as Möbius inversion.

For any finite ring $R$ we denote by $R^{\times}$the group of units of $R$. An application of Möbius inversion pertinent to this paper is then given by the following.

Example 2.5 Let $R$ be a finite ring and let $\mu$ denote the Möbius function on the poset $\{R x \mid$ $x \in R\}$, partially ordered by set inclusion. Let $x \in R$. Then $R^{\times} x$ is the set of all generating elements of of $R x$ and

$$
\left|R^{\times} x\right|=\sum_{R y \leq R x}|R y| \mu(R y, R x)
$$

holds since $|R x|=\sum_{R y \leq R x}\left|R^{\times} y\right|$.

\footnotetext{
${ }^{1} \mathrm{~A}$ poset is a set together with a partial order.
} 


\section{Linear codes and geometries over finite Frobenius rings}

Let $R$ be a finite ring $R$. A left linear code of length $n$ over $R$ is a submodule of ${ }_{R} R^{n}$, which we indicate by writing $C \leq{ }_{R} R^{n}$. We say that $C$ is $k$-generated if $C$ possesses a generating set of $k$ elements. Equivalently, $C$ is the row space of a $k \times n$-matrix over $R$. Note that a $k$-generated code is always $\ell$-generated for all $\ell \geq k$.

A pair of left linear codes $C$ and $C^{\prime}$ over $R$ are said to be isometric relative to the weight function $w$ if there exists a bijective linear $w$-isometry from $C$ onto $C^{\prime}$, i.e. an $R$-linear isomorphism between the codes that preserves $w$. If $w$ is homogeneous and $R$ is a finite Frobenius ring this occurs precisely when the given isomorphism can be extended to a monomial transformation from ${ }_{R} R^{n}$ onto itself (cf. [13]).

For what follows, we need to introduce the notion of projective geometry over a ring. There are various approaches to this in the literature. Here we follow Veldkamp [26, 26.4.1] and adopt the notion of a Barbilian space of a right module $M_{R}$. Let $M^{*}:=\operatorname{Hom}\left(M_{R}, R_{R}\right)$ denote the dual of $M_{R}$. $M^{*}$ forms a left $R$-module by the right action of $R$ on $M$.

The Barbilian space of $\operatorname{Barb}\left(M_{R}\right)$ is defined as the quadruple $(P, H, \mid, \not)$ where

$$
\begin{aligned}
P & :=\left\{x R \mid x \in M \text { with } \varphi x=1 \text { for some } \varphi \in M^{*}\right\} \\
H & :=\left\{R \varphi \mid \varphi \in M^{*} \text { with } \varphi x=1 \text { for some } x \in M\right\} \\
\mid & :=\left\{(R \varphi, x R) \mid(\varphi, x) \in M^{*} \times M \text { with } \varphi x=0\right\} \cap H \times P \\
\not \approx & :=\left\{(R \varphi, x R) \mid(\varphi, x) \in M^{*} \times M \text { with } \varphi x=1\right\}
\end{aligned}
$$

The elements of $P$ are called points, the elements of $H$ are called hyperplanes of $\operatorname{Barb}\left(M_{R}\right)$. Relation | is called incidence, and relation $\not$ is called distant.

A left linear code $C$ may now be viewed as a set of evaluations of an $n$-tuple of linear functionals $\psi_{i}:{ }_{R} R^{k} \longrightarrow{ }_{R} R, i=1, \ldots, n$, for some positive integer $k:$

$$
C=\left\{\left(x \psi_{1}, x \psi_{2}, \ldots, x \psi_{n}\right) \mid x \in R^{k}\right\} .
$$

A generator matrix for $C$ is then given as the $k \times n$ generator matrix $Y=\left(y_{1}\left|y_{2}\right| \ldots \mid y_{n}\right)$, with each $y_{i} \in R^{k}$, if the linear functionals $\varphi_{i}$ correspond to taking the standard inner product $x \psi_{i}:=x \cdot y_{i}$ for each $x \in R^{k}$. Let $\pi_{i}: R^{n} \longrightarrow R, x \mapsto x_{i}$ denote the projection of $R^{n}$ onto its $i$-th coordinate. If all coordinate projections $\pi_{i}$ are surjective, then up to monomial equivalence, the code $C$ is equivalently described by the multiset $\left\{y_{1} R, \ldots, y_{n} R\right\}$ of points in $\operatorname{Barb}\left(R_{R}^{k}\right)$. Such a code will be called a regular code.

For the purposes of this paper, we will always require that the projection of $C$ on any coordinate returns the full ring $R$, so that each column of the generator matrix $Y$ generates a point in $\operatorname{Barb}\left(R_{R}^{k}\right)$. We will also usually require that the points $\left\{y_{1} R, \ldots, y_{n} R\right\}$ are all distinct, in which case we will refer to the generated code as a projective code.

Recall that there exist finite Frobenius rings such that the homogeneous weight of a nonzero element is zero, in which case a linear code over that ring could have nonzero words of weight zero. In most what follows we will rule out such an anomaly of a code by requiring that the code be proper.

More formally we give the following definition. 
Definition 3.1 A linear code $C \leq{ }_{R} R^{n}$ with $k \times n$ generator matrix $Y=\left(y_{1}\left|y_{2}\right| \ldots \mid y_{n}\right)$ is called

(i) regular if $y_{i} R$ is a point of $\operatorname{Barb}\left(R_{R}^{k}\right)$ for each $i \in\{1, \ldots, n\}$,

(ii) projective if $y_{i} R \neq y_{j} R$ for any pair of distinct coordinates $i, j \in\{1, \ldots, n\}$,

(iii) proper if $\mathrm{w}_{\mathrm{hom}}(c)>0$ for every nonzero $c \in C$.

Remark 3.2 A linear code $C \leq{ }_{R} R^{n}$ is projective if and only if the $n$ coordinate projections $\pi_{i}: C \longrightarrow R, c \mapsto c_{i}$ generate distinct cyclic submodules of ${ }_{R} \operatorname{Hom}\left({ }_{R} C,{ }_{R} R\right)$. Hence projectivity of a linear code is a well-defined concept and independent of the particular choice of $Y$.

\section{Two-weight codes over a finite Frobenius ring}

In this section we introduce codes with two non-zero homogeneous weights and make some basic observations. In what follows let $R$ be a finite Frobenius ring, and let $w$ be a homogeneous weight on $R$ of average value $\gamma \neq 0$.

The following can be deduced directly from the homogeneity condition $\mathbf{H 2}$ * It is an immediate extension of a statement given in [3] (cf. also [13]).

Lemma 4.1 If $C \leq{ }_{R} R^{n}$ is a linear code over $R$ then for all $i=1, \ldots, n$ there holds

$$
\sum_{c \in C} w\left(c_{i}\right)= \begin{cases}\gamma|C| & \text { if } \pi_{i}(C) \neq 0 \\ 0 & \text { otherwise }\end{cases}
$$

Definition 4.2 A proper linear code $C \leq{ }_{R} R^{n}$ is called a two-weight code, if $w$ takes exactly two nonzero values $w_{1}$ and $w_{2}$ on $C$.

If the normalized homogeneous weight $\mathrm{w}_{\text {hom }}$ on $R$ is strictly positive, i.e. if $\mathrm{w}_{\text {hom }}(x)>0$ for all $0 \neq x \in R$, then all codes over $R$ are proper.

The class of finite Frobenius rings where the normalised homogeneous weight is not strictly positive was characterised in [20, Th. 1]:

Proposition 4.3 The normalized homogeneous weight $\mathrm{w}_{\text {hom }}$ on a finite Frobenius ring $R$ is positive definite if and only if $R$ has at most one two-sided ideal of cardinality 2 .

Since the sum of the weights in a nonzero coordinate of $C$ is fixed and given by $\gamma|C|$, we have the following system of equations.

Observation 4.4 Let $C$ be a two-weight code of length $n$ with weights $w_{1}$ and $w_{2}$. Let $b_{1}$ denote the number of codewords of weight $w_{1}$ and $b_{2}$ the number of codewords of weight $w_{2}$. If $\pi_{i}(C) \neq\{0\}$ for all $i=1, \ldots, n$ then

$$
b_{1} w_{1}+b_{2} w_{2}=\gamma n|C| \text { and } b_{1}+b_{2}=|C|-1 \text {. }
$$


This system of equations determines the relationship between the $b_{i},|C|, w_{i}$ and $n$. In particular, it shows that the values $b_{i}$ are uniquely determined by $n,|C|, w_{1}$ and $w_{2}$. We prefer to rewrite the above system in the following more convenient matrix form:

$$
\left[\begin{array}{cc}
w_{1} & w_{2} \\
1 & 1
\end{array}\right] \cdot\left[\begin{array}{l}
b_{1} \\
b_{2}
\end{array}\right]=\left[\begin{array}{c}
\gamma n|C| \\
|C|-1
\end{array}\right] .
$$

Given a linear code $C \leq{ }_{R} R^{n}$ and a left ideal $I$ of $R$, for each $i \in\{1, \ldots, n\}$ we write $C(i, I)$ to denote the left $R$-submodule of $C$ defined by

$$
C(i, I):=\left\{c \in C \mid \pi_{i}(c) \in I\right\} .
$$

Lemma 4.5 Let $C \leq{ }_{R} R^{n}$ be a linear code and let $I$ be a left ideal of $R$. Then for each $i \in\{1, \ldots, n\}$,

$$
|C(i, I)|=\frac{|I|}{\left|I+\pi_{i}(C)\right|}|C| .
$$

In particular, if $\pi_{i}(C)=R$ then

$$
|C(i, I)|=\frac{|I|}{|R|}|C| .
$$

Proof: First note that $|C(i, I)|=\sum_{r \in I}\left|\left\{c \in C \mid \pi_{i}(c)=r\right\}\right|$. Now if $r$ is an element in $I \cap \pi_{i}(C)$ then $r$ occurs in the $i$-th position of exactly $\left|\operatorname{Ker}\left(\pi_{i}\right) \cap C\right|$ words of $C$. Then, summing over all $r$ in $I$, we obtain

$$
|C(i, I)|=\left|I \cap \pi_{i}(C)\right| \cdot\left|\operatorname{Ker}\left(\pi_{i}\right) \cap C\right| .
$$

A simple homomorphism argument completes the proof.

An immediate consequence of Lemma 4.5 is that if $C$ is regular, then the expression $|C(i, I)|$ depends on $C$ and $I$, certainly, but is independent of the chosen coordinate $i$.

Lemma 4.6 Given a regular projective code $C \leq{ }_{R} R^{n}$ and any pair $i, j \in\{1, \ldots, n\}$ of distinct coordinates, there exists a codeword $c=\left(c_{1}, \ldots, c_{n}\right) \in C$ with $c_{i}=0$ and $c_{j} \neq 0$.

Proof: Let $C$ have $k \times n$ generator matrix $\left(y_{1}|\cdots| y_{n}\right)$. Since $C$ is regular, $\pi_{i}(C)=y_{i} R$ for each $i$ and $C / \operatorname{Ker} \pi_{i}=y_{i} R$. Since $C$ is projective the $y_{i} R$ are distinct and hence $C \cap \operatorname{Ker} \pi_{i} \neq C \cap \operatorname{Ker} \pi_{j}$ for $i \neq j$. Furthermore, since $C$ is regular, from Lemma 4.5 $\left|C \cap \operatorname{Ker} \pi_{i}\right|=\left|C \cap \operatorname{Ker} \pi_{j}\right|=\frac{|C|}{|R|}$. Now, the result follows.

Given a two-weight code $C$ with weights $w_{1}$ and $w_{2}$ define

$$
C_{1}:=\left\{c \in C \mid w(c)=w_{1}\right\}, \text { and } C_{2}:=\left\{c \in C \mid w(c)=w_{2}\right\} .
$$

For an arbitrary subset $T \subseteq R$ we define

$$
b_{1}(i, T):=\left|\left\{c \in C_{1} \mid \pi_{i}(c) \in T\right\}\right|, \text { and } b_{2}(i, T):=\left|\left\{c \in C_{2} \mid \pi_{i}(c) \in T\right\}\right| .
$$

Our next goal is to show that $b_{1}(i, I)$ (and hence $b_{2}(i, I)$ ) does not depend on the chosen coordinate $i$, provided $C$ is regular and projective and $I$ is a left ideal of $R$.

Using Lemma 4.5 we extend Observation 4.4 in the case of regular projective codes as follows. 
Proposition 4.7 Let $C \leq{ }_{R} R^{n}$ be a regular projective two-weight code with weights $w_{1}$ and $w_{2}$. For each $i \in\{1, \ldots, n\}$ and $I \leq{ }_{R} R$ then

$$
\left[\begin{array}{cc}
w_{1} & w_{2} \\
1 & 1
\end{array}\right] \cdot\left[\begin{array}{l}
b_{1}(i, I) \\
b_{2}(i, I)
\end{array}\right]=\left[\begin{array}{l}
\gamma n \frac{|I|}{|R|}|C| \\
\frac{|I|}{|R|}|C|-1
\end{array}\right]
$$

if $I \neq 0$, and

$$
\left[\begin{array}{cc}
w_{1} & w_{2} \\
1 & 1
\end{array}\right] \cdot\left[\begin{array}{l}
b_{1}(i, 0) \\
b_{2}(i, 0)
\end{array}\right]=\left[\begin{array}{c}
\gamma(n-1) \frac{|C|}{|R|} \\
\frac{|C|}{|R|}-1
\end{array}\right]
$$

provided $I=0$. In particular, the values $b_{1}(i, I)$ and $b_{2}(i, I)$ are independent of $i$ for all nonzero $I \leq{ }_{R} R$.

Proof: We count in two ways the total homogeneous weight of the linear subcode $C(i, I)$ of $C$. From Lemma 4.1 we have

$$
w_{1} b_{1}(i, I)+w_{2} b_{2}(i, I)=\sum_{c \in C(i, I)} w(c)=\sum_{j=1}^{n} \sum_{c \in C(i, I)} w\left(c_{j}\right)=\gamma s|C(i, I)|,
$$

where $s:=\left|\left\{j \mid \pi_{j}(C(i, I)) \neq 0\right\}\right|$. Since $C$ is regular and projective, from Lemma 4.6 we have $\pi_{j}(C(i,\{0\})) \neq 0$ for $i \neq j$. Hence, $s=n-1$ or $s=n$ depending on whether $I=0$ or $I \neq 0$, respectively. Using Lemma 4.5 we obtain $|C(i, I)|=\frac{|I|}{|R|}|C|$ for any $I$, which yields the result in both cases.

In light of the fact that the numbers $b_{1}(i, I)$ and $b_{2}(i, I)$ are independent of the choice of $i$ for a given regular projective two-weight code, we will refer to these magnitudes simply as $b_{1}(I)$ and $b_{2}(I)$. Of course, $b_{1}(R)=b_{1}$ and $b_{2}(R)=b_{2}$ as introduced in Observation 4.4.

\section{Two-weight codes and strongly regular graphs}

We will now draw connections between two-weight codes and strongly regular graphs. In particular, we show that every linear code over a finite Frobenius ring with exactly two non-zero homogeneous weights renders a strongly regular graph.

Definition 5.1 A simple graph $\Gamma=(V, E)$ with vertex set $V$ and edge set $E$ is called strongly regular with parameters $(N, K, \lambda, \mu)$ if:

(i) $\Gamma$ has $N$ vertices and is neither empty nor complete,

(ii) $\Gamma$ is regular of degree $K$, and

(iii) Every adjacent pair of vertices $v, v^{\prime} \in V$ has exactly $\lambda$ common neighbours in $V$

(iv) Every non-adjacent pair of vertices $v, v^{\prime} \in V$ has exactly $\mu$ common neighbours in $V$.

Strongly regular graphs are well-studied and are equivalent to symmetric association schemes with 2 classes. Corresponding to any strongly regular graph $\Gamma$ is a 3 -dimensional Bose-Mesner algebra 
spanned by the $N \times N$ identity matrix $I$, the all-one matrix $J$ and the adjacency matrix $A$ of $\Gamma$. The reader is referred to [6, 7, 19] for properties of such graphs and their relations to other combinatorial objects. The complement of a strongly regular graph with parameters $(N, K, \lambda, \mu)$ is also strongly regular and has parameters $(N, N-K-1, N-2 K+\mu-2, N-2 K+\lambda)$. A strongly regular graph is said to be trivial if either $\Gamma$ or its complement is a disjoint union of complete graphs (of the same size). A strongly regular graph $\Gamma$ is called nontrivial if and only if its parameters satisfy the condition $0<\mu<K$.

The parameters of a strongly regular graph must satisfy certain feasibility conditions. For example, if $\Gamma=(V, E)$ is strongly regular with parameters $(N, K, \lambda, \mu)$ then a simple counting argument shows that $K(K-\lambda-1)=\mu(N-K-1)$. The parameters $N, K, \lambda, \mu$ are called feasible if they satisfy well-known feasibility conditions, and an important aspect of the theory of strongly regular graphs is to establish the existence of a strongly regular graph for a given set of feasible parameters.

Let $G$ be an additive abelian group and let $D$ be a subset of $G$ such that $-d \in D$ for each $d \in D$ and $0 \notin D$. The Cayley graph of $G$ with respect to $D$ is given by the graph $\Gamma=(G, E)$ with vertex set $G$ and edge set $E=\{(g, d+g) \mid g \in G, d \in D\}$. Then $\Gamma$ is regular of degree $|D|$ and is strongly regular if and only if $D$ is a so-called (regular) partial difference set (see [6, 22]).

In this section we consider this construction for the particular case where $G$ is a submodule of ${ }_{R} R^{n}$ (i.e. an $R$-linear code) and $D$ is a subset of $G$ (in fact the set of words of a constant weight subcode of $G$ ). We will show that if $G$ is a regular projective two-weight code, and $D$ consists of the codewords of a given weight, say $w_{1}$, then the graph $\Gamma=(G, E)$ defined above is strongly regular.

Lemma 5.2 Let $C \leq{ }_{R} R^{n}$ be a regular projective two-weight code with weights $w_{1}$ and $w_{2}$, and let $C_{1}$ and $C_{2}$ be as defined in Equation (11). Then for each $i \in\{1, \ldots, n\}$ we have

$$
\sum_{x \in C_{1}} w\left(x_{i}\right)=\frac{b_{1} w_{1}}{n} \quad \text { and } \quad \sum_{x \in C_{2}} w\left(x_{i}\right)=\frac{b_{2} w_{2}}{n} .
$$

Proof: We need only show $\sum_{x \in C_{1}} w\left(x_{i}\right)$ is independent of $i$, since in that case we find that the total homogeneous weight of the words of weight $w_{1}$ in $C$ (which is given by $w_{1} b_{1}$ ) equals $n \sum_{x \in C_{1}} w\left(x_{i}\right)$. As in Example 2.5, first observe that

$$
b_{1}\left(i, R^{\times} x\right)=\sum_{R y \leq R x} b_{1}(i, R y) \mu(R y, R x)
$$

by Möbius inversion. By Proposition 4.7, these magnitudes clearly do not depend on the chosen coordinate $i$, and so we can write $b_{1}\left(i, R^{\times} x\right)=b_{1}\left(R^{\times} x\right)$ for all $i$. Finally we obtain

$$
\sum_{x \in C_{1}} w\left(x_{i}\right)=\sum_{R y \leq R} w(y) b_{1}\left(R^{\times} y\right)
$$

which again will not depend on the choice of $i$. This completes the proof for both claims.

Proposition 5.3 Let $C \leq{ }_{R} R^{n}$ be a regular projective two-weight code with weights $w_{1}$ and $w_{2}$. Then

$$
\sum_{x \in C_{1}} w(x-c)=\gamma n b_{1}\left[1-\left(1-\frac{w_{1}}{\gamma n}\right)\left(1-\frac{w(c)}{\gamma n}\right)\right]
$$


for every $c \in C$.

Proof: Using Proposition 2.3 we compute $\sum_{x \in C_{1}} w(x-c)$ as follows.

$$
\begin{aligned}
\sum_{x \in C_{1}} w(x-c) & =\sum_{x \in C_{1}} \sum_{i=1}^{n} w\left(x_{i}-c_{i}\right) \\
& =\gamma \sum_{x \in C_{1}} \sum_{i=1}^{n}\left[1-\frac{1}{\left|R^{\times}\right|} \sum_{u \in R^{\times}} \chi\left(u\left[x_{i}-c_{i}\right]\right)\right] \\
& =\gamma n b_{1}-\gamma \sum_{x \in C_{1}} \sum_{i=1}^{n} \frac{1}{\left|R^{\times}\right|} \sum_{u \in R^{\times}} \chi\left(-u c_{i}\right) \chi\left(u x_{i}\right) \\
& =\gamma n b_{1}-\gamma \sum_{i=1}^{n} \frac{1}{\left|R^{\times}\right|} \sum_{u \in R^{\times}} \chi\left(-u c_{i}\right)\left[\sum_{x \in C_{1}} \chi\left(u x_{i}\right)\right] .
\end{aligned}
$$

Observing that as $u C_{1}=C_{1}$ for all $u \in R^{\times}$, we get

$$
\sum_{x \in C_{1}} \chi\left(u x_{i}\right)=\sum_{x \in C_{1}} \chi\left(x_{i}\right)=\frac{1}{\left|R^{\times}\right|} \sum_{v \in R^{\times}} \sum_{x \in C_{1}} \chi\left(v x_{i}\right)=\sum_{x \in C_{1}} \frac{1}{\left|R^{\times}\right|} \sum_{v \in R^{\times}} \chi\left(v x_{i}\right),
$$

and so we may write

$$
\begin{aligned}
\sum_{x \in C_{1}} w(x-c) & =\gamma n b_{1}-\gamma \sum_{i=1}^{n}\left[\frac{1}{\left|R^{\times}\right|} \sum_{u \in R^{\times}} \chi\left(-u c_{i}\right)\right] \sum_{x \in C_{1}}\left[\frac{1}{\left|R^{\times}\right|} \sum_{v \in R^{\times}} \chi\left(v x_{i}\right)\right] \\
& =\gamma n b_{1}-\gamma \sum_{i=1}^{n}\left(1-\frac{w\left(c_{i}\right)}{\gamma}\right) \sum_{x \in C_{1}}\left(1-\frac{w\left(x_{i}\right)}{\gamma}\right) .
\end{aligned}
$$

Using Lemma 5.2 we can rewrite the latter expression as

$$
\sum_{x \in C_{1}}\left(1-\frac{w\left(x_{i}\right)}{\gamma}\right)=b_{1}-\frac{b_{1} w_{1}}{\gamma n}=b_{1}\left(1-\frac{w_{1}}{\gamma n}\right),
$$

which is clearly independent of the coordinate $i$, so that we have

$$
\begin{aligned}
\sum_{x \in C_{1}} w(x-c) & =\gamma n b_{1}-\gamma b_{1}\left(1-\frac{w_{1}}{\gamma n}\right) \sum_{i=1}^{n}\left(1-\frac{w\left(c_{i}\right)}{\gamma}\right) \\
& =\gamma n b_{1}-\gamma n b_{1}\left(1-\frac{w_{1}}{\gamma n}\right)\left(1-\frac{w(c)}{\gamma n}\right) \\
& =\gamma n b_{1}\left[1-\left(1-\frac{w_{1}}{\gamma n}\right)\left(1-\frac{w(c)}{\gamma n}\right)\right] .
\end{aligned}
$$

This completes the proof.

This result allows us to set up a new system of equations, as indicated below. 
Corollary 5.4 Let $C \leq{ }_{R} R^{n}$ be a regular projective two-weight code with weights $w_{1}$ and $w_{2}$, and let $C_{1}$ and $C_{2}$ be defined as in Equation (10). Given $c_{1} \in C_{1}$ and $c_{2} \in C_{2}$, let

$$
d_{i}\left(c_{1}\right)=\left|\left\{x \in C_{1} \mid w\left(x-c_{1}\right)=w_{i}\right\}\right| \quad \text { and } \quad e_{i}\left(c_{2}\right)=\left|\left\{x \in C_{1} \mid w\left(x-c_{2}\right)=w_{i}\right\}\right|
$$

for $i \in\{1,2\}$. Then

$$
\left[\begin{array}{cc}
w_{1} & w_{2} \\
1 & 1
\end{array}\right] \cdot\left[\begin{array}{l}
d_{1}\left(c_{1}\right) \\
d_{2}\left(c_{1}\right)
\end{array}\right]=\left[\begin{array}{c}
D \\
b_{1}-1
\end{array}\right] \quad \text { and } \quad\left[\begin{array}{cc}
w_{1} & w_{2} \\
1 & 1
\end{array}\right] \cdot\left[\begin{array}{l}
e_{1}\left(c_{2}\right) \\
e_{2}\left(c_{2}\right)
\end{array}\right]=\left[\begin{array}{l}
E \\
b_{1}
\end{array}\right]
$$

where $D=\gamma n b_{1}\left[1-\left(1-\frac{w_{1}}{\gamma n}\right)^{2}\right]$ and $E=\gamma n b_{1}\left[1-\left(1-\frac{w_{1}}{\gamma n}\right)\left(1-\frac{w_{2}}{\gamma n}\right)\right]$. In particular, the numbers $d_{i}\left(c_{1}\right), e_{i}\left(c_{2}\right)$ do not depend on the choice of $c_{1} \in C_{1}$ respectively $c_{2} \in C_{2}$.

Corollary 5.4 says that in a linear regular projective two-weight code over a finite Frobenius ring, the number of words of weight $w_{1}$ at distance $w_{1}$ from a word of weight $w_{1}$ is constant and given by $d_{1}=d_{1}\left(c_{1}\right)$, and the number of words of weight $w_{1}$ at distance $w_{1}$ from a word of weight $w_{2}$ is constant and given by $e_{1}=e_{1}\left(c_{2}\right)$. The linearity of $C$ then immediately gives the following result.

Theorem 5.5 Let $C \leq{ }_{R} R^{n}$ be a regular projective two-weight code over a finite Frobenius ring $R$ with normalized homogeneous weights $w_{1}$ and $w_{2}$. Then the graph $\Gamma(C):=(C, E)$ with vertex set $C$ and edge set $E:=\left\{\{x, y\} \mid x, y \in C\right.$ with $\left.w(x-y)=w_{1}\right\}$ is strongly regular. Its parameters are $(N, K, \lambda, \mu)$, where

$$
\begin{aligned}
& N=|C| \\
& K=\frac{\left(n-w_{2}\right)|C|+w_{2}}{w_{1}-w_{2}}, \\
& \lambda=\frac{n K\left[1-\left(1-\frac{w_{1}}{n}\right)^{2}\right]+w_{2}(1-K)}{w_{1}-w_{2}}, \\
& \mu=\frac{n K\left[1-\left(1-\frac{w_{1}}{n}\right)\left(1-\frac{w_{2}}{n}\right)\right]-w_{2} K}{w_{1}-w_{2}} .
\end{aligned}
$$

We now give some simple examples illustrating the use of Theorem 5.5. Further examples will be presented in the next section.

Example 5.6 The code $C=\left\{x \in \mathbb{Z}_{4}^{3} \mid x_{1}+x_{2}+x_{3}=0\right\}$, i.e. the parity-check code of length 3 over $\mathbb{Z}_{4}$, is a regular projective two-weight code with nonzero Lee weights $w_{1}=2, w_{2}=4$ and frequencies $b_{1}=6, b_{2}=9$. The constant weight subcodes of $C$ are

$$
C_{1}=\{130,310,103,301,013,031\} \quad \text { and } \quad C_{2}=\{112,332,121,323,211,233,220,202,022\} .
$$

By Theorem 5.5, the graph $\Gamma(C)$ obtained from the codewords in $C_{1}$ is strongly regular with parameters $(16,6,2,2)$. It is easily checked that the induced subgraph on $C_{1}$ (the neighborhood of the vertex 000$)$ is a 6 -cycle. Hence $\Gamma(C)$ is isomorphic to the Shrikhande graph (cf. [7, Ex. 4.6]). 
The other $(16,6,2,2)$ strongly regular graph, the square lattice graph $\mathrm{L}_{2}(4)$, is obtained by taking instead of $\mathbb{Z}_{4}$ one of the rings $\mathbb{F}_{4}$ or $\mathbb{F}_{2}[X] /\left(X^{2}\right)$.

Now consider fourth ring of order $4, \mathbb{F}_{2} \oplus \mathbb{F}_{2}$, and replace $\mathbb{Z}_{4}$ with it in the above. The resulting code is not proper and the constant weight subcodes containing words of weight 0 and 4 are

$$
C_{1}=\{o o o, o c c, c o c, c c o\} \quad \text { and } \quad C_{2}:=\{o a a, a o a, a a o, o b b, b o b, b b o, a b c, a b c, b a c, b c a, c a b, c b a\}
$$

where $o=00, a=10, b=01$ and $c=11$. Although the code is not proper, it still gives the (trivial) strongly regular with parameters $(16,3,2,0)$.

\section{Constructions}

The constructions given here require that the reader be familiar with the subclass of Frobenius rings called chain rings. A ring $R$ is called a left chain ring, if the set of its left ideals forms a chain by set inclusion. If $R$ is finite it can be seen that also the set of all right ideals of $R$ forms a chain, and hence we refer to these rings simply as finite chain rings.

The unique maximal ideal $\operatorname{Rad}(R)$ of a finite chain ring $R$ is principal, and hence generated by an element $\theta \in R$. The nilpotency index of $\theta$ is then called the length of $R$, and our first pair of examples assume that $R$ is of length 2 . Observe that $F:=R / \operatorname{Rad}(R)$ is a finite field, of size $q$, say, and $\operatorname{Rad}(R)$ has $q$ elements.

On the set of points of $\operatorname{Barb}\left(R_{R}^{2}\right)$ we define the neighbourhood relation $\sim$ by $x R \sim y R$ if and only if $\nu(x R)=\nu(y R)$ where $\nu: R^{2} \longrightarrow \mathbb{F}_{q}^{2}$ is the natural semilinear epimorphism defined by $x \mapsto x+\operatorname{Rad}\left(R_{R}^{2}\right)$. This relation is an equivalence relation which has $q+1$ equivalence classes, and each of these classes consists of $q$ points.

Proposition 6.1 Let $R$ be a finite chain ring of length 2 with a $q$-element residual field. Let $1 \leq s \leq q$ be an integer. Let $Y$ be a $2 \times s(q+1)$ matrix whose columns generate $s$ distinct elements of each equivalence class of $\sim$ given above. Then $Y$ generates an $[s(q+1), 2]$ two-weight code with weights

$$
w_{1}=\frac{q(q s-1)}{q-1} \quad \text { and } w_{2}=\frac{q^{2} s}{q-1} .
$$

Proof: Words of the generated code $C$ are of the form $x Y$ where $x \in R^{2}$. If $x$ is a unimodular element (i.e. there is $z \in R$ with $x_{1} z_{1}+x_{2} z_{2}=1$ ) then there are exactly $q s$ columns $z$ in $Y$ for which $x z \in R^{\times}$. For each of the remaining $s$ columns $z$ of $Y$ we have $x z \in \operatorname{Rad}(R)$, where there is at most one column $z$ with $x z=0$. For this reason we have

$$
\mathrm{w}_{\text {hom }}(x Y)= \begin{cases}q s+(s-1) \frac{q}{q-1} & =\frac{q(q s-1)}{q-1}, \text { or } \\ q s+s \frac{q}{q-1} & =\frac{q^{2} s}{q-1} .\end{cases}
$$

If $x$ is non-zero, but not unimodular, then $x=\theta x^{\prime}$ for some unimodular $x^{\prime} \in R^{2}$ (and where $\theta$ is a generator of $\operatorname{Rad}(R))$. The codeword in question is given by $\theta x^{\prime} Y$. For all columns of $Y$ generating points in the same equivalence class of $\sim$ the expression $\theta x^{\prime} Y$ is constant, and 
it vanishes on exactly one class. This means that $w_{H}(x Y)=q s$, and for this reason we find $\mathrm{w}_{\text {hom }}(x Y)=q s \frac{q}{q-1}$. $C$ is now proper because $\mathrm{w}_{\text {hom }}$ is strictly positive. All in all we have hence proven the claim.

Corollary 6.2 The code $C$ in the previous proposition determines a strongly regular graph with parameters

$$
N=q^{4}, \quad K=s\left(q^{3}-q\right), \quad \lambda=q^{2}\left(1+s^{2}\right)-3 s q, \quad \mu=s q(s q-1) .
$$

For example, let $R=\mathbb{Z}_{9}$. The ring $R$ has maximal ideal $3 \mathbb{Z}_{9}$, which has 3 elements. Now the set of points in $\operatorname{Barb}\left(\mathbb{Z}_{9}^{2}\right)$ has $q+1=4$ distinct equivalence classes, and each class contains 3 points. Choose $s$ points from each class, for $s=1,2$, or 3 and form a generator matrix for a $\mathbb{Z}_{9}$-code with two homogeneous weights $w_{1}, w_{2}$. The table below gives the parameters of the codes constructed as described before, and the parameters of the corresponding strongly regular graph.

\begin{tabular}{|cccc|cccc|}
\hline$n$ & $k$ & $w_{1}$ & $w_{2}$ & $N$ & $K$ & $\lambda$ & $\mu$ \\
\hline 4 & 2 & 3 & 4.5 & 81 & 24 & 9 & 6 \\
\hline 8 & 2 & 7.5 & 9 & 81 & 48 & 27 & 30 \\
\hline 12 & 2 & 12 & 13.5 & 81 & 72 & 63 & 72 \\
\hline
\end{tabular}

The $[12,2]$ code over $\mathbb{Z}_{9}$ with homogeneous weights 12 and 13.5 is the simplex code, and gives a trivial strongly regular graph.

Strongly regular graphs with parameters $(81,24,9,6)$ can also be constructed from the block graph of an orthogonal array $\mathrm{OA}(9,3)$, or a projective ternary $[12,4]$ code with Hamming weights 6 and 9. The complement of such a graph has parameters $(81,56,37,42)$ and may also be constructed from the block graph of $\mathrm{OA}(9,7)$.

Strongly regular graphs with parameters $(81,48,27,30)$ are constructed from the block graph of an orthogonal array $\mathrm{OA}(9,6)$. The corresponding complements have parameters $(81,32,13,12)$ and may be constructed from a projective ternary [16,4] code with Hamming weights 9 and 12 or from the block graph of an orthogonal array $\mathrm{OA}(9,4)$.

The next example needs some preparation. Let $R=\mathbb{F}_{q}[X ; \sigma] /\left(X^{2}\right)$ for some $\sigma \in \operatorname{Aut}\left(\mathbb{F}_{q}\right)$ be a truncated skew polynomial ring. There is a natural embedding $\operatorname{PGL}\left(3, \mathbb{F}_{q}\right) \stackrel{e}{\longrightarrow} \operatorname{PGL}(3, R)$. If $p$ is a point of $\operatorname{Barb}\left(R_{R}^{3}\right)$ then the orbit of $p$ under an ( $e$-embedded) Singer cycle of $\operatorname{PGL}\left(2, \mathbb{F}_{q}\right)$ is a $q^{2}+q+1$ element point set $K$ that shares exactly 1 or $q+1$ points with every hyperplane of $\operatorname{Barb}\left(R_{R}^{3}\right)$.

Proposition 6.3 Let $R$ be the above truncated skew polynomial ring, and let $Y$ be a $3 \times\left(q^{2}+\right.$ $q+1)$-matrix over $R$ whose columns are generating the points of $K$ as defined above. Then $Y$ generates a $\left[q^{2}+q+1,3\right]$ two-weight code with weights

$$
w_{1}=\frac{q^{3}}{q-1} \quad \text { and } w_{2}=q^{2}
$$


Proof: We will only sketch the proof. Words of the generated code are of the form $x Y$ where $x \in R^{3}$. Similar to the proof of Proposition 6.1 we can show that if $x$ is unimodular, then

$$
\mathrm{w}_{\text {hom }}(x Y)=\frac{q^{3}}{q-1} \quad \text { or } \mathrm{w}_{\text {hom }}(x Y)=q^{2} .
$$

If $x$ is a non-zero element of $\operatorname{Rad}\left({ }_{R} R^{3}\right)$ then $\mathrm{whom}_{\mathrm{h}}(x Y)=\frac{q^{3}}{q-1}$. This completes the proof.

Corollary 6.4 The code $C$ in the previous proposition determines a strongly regular graph with parameters

$$
N=q^{6}, \quad K=q^{4}-q, \quad \lambda=q^{3}+q^{2}-3 q, \quad \mu=q^{2}-q .
$$

For example, for $p=2$ we get the table

\begin{tabular}{|cccc|cccc|}
\hline$n$ & $k$ & $w_{1}$ & $w_{2}$ & $N$ & $K$ & $\lambda$ & $\mu$ \\
\hline 7 & 3 & 4 & 8 & 64 & 14 & 6 & 2 \\
\hline 21 & 3 & 16 & $64 / 3$ & 4096 & 252 & 68 & 12 \\
\hline 73 & 3 & 64 & $512 / 7$ & 262144 & 4088 & 552 & 56 \\
\hline
\end{tabular}

The strongly regular graph with parameters $(64,14,6,2)$ is unique, and may also be constructed from a projective binary $[14,6]$ code with Hamming weights 4 and 8 .

Our last example again needs further preparation. In some way it combines the ideas of the first two examples.

First recall that if $\mathbb{F}_{q}$ is the finite field of $q$ elements, then in the projective plane $\mathrm{PG}\left(F_{q}^{3}\right)$ there is a bijection $\sigma$ between the set of points and the set of lines, such that $p \in \sigma(p)$ for all points. $\sigma$ might for example be chosen as the orbit of a flag $(p, \ell)$ under a Singer cycle of the plane.

Let now $R$ be a chain ring with residue field $F_{q}$. Similar to what we introduced earlier we have an equivalence relation $\sim$ on the set of all points of $\operatorname{Barb}\left(R_{R}^{3}\right)$ defined by $x R \sim y R$ if and only if $\nu(x R)=\nu(y R)$. Here $\nu: R^{3} \longrightarrow F_{q}^{3}$ is the natural semilinear epimorphism with $x \mapsto x+\operatorname{Rad}\left(R^{3}\right)_{R}$. This neighbourhood relation has exactly $q^{2}+q+1$ classes. Let $p$ be a point, and let $h$ be a hyperplane in $\operatorname{Barb}\left(R_{R}^{3}\right)$. We define

$$
[p]_{h}:=\{g \mid g \sim p \text { and } g \mid h\},
$$

and call it a line segment, provided it is non-empty. We will call $\nu(h)$ the direction of the line segment.

It is now possible to compose a $q\left(q^{2}+q+1\right)$-element set $K$ of complemented free points of $\operatorname{Barb}\left(R_{R}^{3}\right)$ that meets every neightbourhood class $[p]$ in a line segment in such a way that the occuring directions are all pairwise distinct. This is accomplished using the bijection $\sigma$ introduced above.

Proposition 6.5 Let $Y$ be a $3 \times s q\left(q^{2}+q+1\right)$ matrix whose columns generate the elements of the set $K$ in $\operatorname{Barb}\left(R_{R}^{3}\right)$. The code generated by $Y$ over $R$ is a two-weight code with parameters given by

$$
n=q\left(q^{2}+q+1\right), \quad k=3, \quad w_{1}=\frac{q^{4}-q^{2}}{q-1}, \quad w_{2}=\frac{q^{4}}{q-1},
$$


and determines a strongly regular graph with parameters

$$
N=q^{6}, \quad K=q^{5}-q^{2}, \quad \lambda=q^{4}+q^{3}-3 q^{2}, \quad \mu=q^{2}\left(q^{2}-1\right) .
$$

Remark 6.6 This construction can be generalised to sets $K$ containing $s$ line segments of the same direction from each equivalence class of points, where $1 \leq s \leq q$. Such a set $K$ will have either $w q+q$ or $s q$ points in common with every hyperplane.

The two-weight code will then have parameters given by

$$
n=s q\left(q^{2}+q+1\right), \quad k=3, \quad w_{1}=\frac{s q^{4}-q^{2}}{q-1}, \quad w_{2}=\frac{s q^{4}}{q-1},
$$

and the induced strongly regular graph will have parameters

$$
N=q^{6}, \quad K=s\left(q^{5}-q^{2}\right), \quad \lambda=s^{2} q^{4}+q^{3}-3 s q^{2}, \quad \mu=s q^{2}\left(s q^{2}-1\right) .
$$

For example, for $q=2$ we get codes and graphs with parameters

\begin{tabular}{|cccc|cccc|}
\hline$n$ & $k$ & $w_{1}$ & $w_{2}$ & $N$ & $K$ & $\lambda$ & $\mu$ \\
\hline 14 & 3 & 12 & 16 & 64 & 28 & 12 & 12 \\
\hline 28 & 3 & 28 & 32 & 64 & 56 & 48 & 56 \\
\hline
\end{tabular}

whereas for $q=3$ we get codes and graphs with parameters

\begin{tabular}{|cccc|cccc|}
\hline$n$ & $k$ & $w_{1}$ & $w_{2}$ & $N$ & $K$ & $\lambda$ & $\mu$ \\
\hline 39 & 3 & 36 & 40.5 & 729 & 234 & 81 & 72 \\
\hline 78 & 3 & 76.5 & 81 & 729 & 468 & 297 & 306 \\
\hline 117 & 3 & 117 & 121.5 & 729 & 702 & 675 & 702 \\
\hline
\end{tabular}

\section{Acknowledgement}

The authors feel indebted to the reviewers for a variety of remarks and corrections which helped to improve the quality of the published paper.

\section{References}

[1] M. Aigner, Combinatorial theory, Springer, Berlin, Heidelberg, New York, 1997.

[2] E. Byrne, K. O'Flaherty, "Linear codes over $\mathbb{Z}_{4}$ with two Lee weights", preprint.

[3] I. Constantinescu and W. Heise, A metric for codes over residue class rings of integers, Problemy Peredachi Informatsii 33, no. 3, 22-28, 1997.

[4] P. Delsarte, "Two-weight linear codes and strongly regular graphs", Report R160, MBLE Res. Labs., Brussels, 1971. 
[5] P. Delsarte, "Weights of linear codes and strongly regular normed spaces", Discrete Math., 3, pp. 47-64, 1972.

[6] R. Calderbank, W. M. Kantor, "The geometry of two-weight codes", Bulletin of the London Mathematical Society, 18, pp. 97-122, 1986.

[7] P. J. Cameron, J. H. van Lint, Designs, Graphs, Codes and their Links, Cambridge University Press, 1991.

[8] P. Camion, "Codes and association schemes", Handbook of Coding Theory, Vol. II, Elsevier Science, North-Holland, 1998.

[9] C. Faith, Lectures on injective modules, Lecture Notes in Mathematics, Vol. 49, SpringerVerlag, 1967.

[10] C.Faith, Algebra II: Ring Theory, Grundlehren der mathematischen Wissenschaften, Vol 191, Springer-Verlag, 1976.

[11] M. Greferath and S. E. Schmidt. Gray isometries for finite chain rings. IEEE Transactions on Information Theory, 45(7):2522-2524, Nov. 1999.

[12] Marcus Greferath, Alexandr Nechaev, and Robert Wisbauer, Finite quasi-Frobenius modules and linear codes, J. Algebra Appl. 3 (2004), no. 3, 247-272.

[13] M. Greferath and S. E. Schmidt. Finite-ring combinatorics and MacWilliams' equivalence theorem. Journal of Combinatorial Theory, Series A, 92:17-28, 2000.

[14] A. Hammons, P. Kumar, A. Calderbank, N. Sloane, P. Sole, "The $\mathbb{Z}_{4}-$ linearity of Kerdock, Preparata, Goethals, and related codes", IEEE Trans. Inform. Theory, Vol. 40, No. 2, pp. 301-319, 1994.

[15] W. Heise and T. Honold. Homogeneous and egalitarian weights on finite rings. In Proceedings of the Seventh International Workshop on Algebraic and Combinatorial Coding Theory (ACCT2000), pages 183-188, Bansko, Bulgaria, 2000.

[16] T. Honold, I. Landjev, Linearly representable codes over chain rings. Abhandlungen aus dem mathematischen Seminar der Universität Hamburg, 69:187-203, 1999.

[17] T. Honold, I. Landjev, Linear codes over finite chain rings. Electronic Journal of Combinatorics, 7(\#R11), 2000.

[18] T. Y. Lam, Lectures on Modules and Rings, Graduate Texts in Mathematics, Vol. 189, SpringerVerlag, 1999.

[19] J. H. van Lint, R. M. Wilson, A Course in Combinatorics, Cambridge Univ. Press, 1998

[20] T. Honold, A. Nechaev, Weighted modules and representations of codes. (Russian) Problemy Peredachi Informatsii 35 (1999), no. 3, 18-39; translation in Problems Inform. Transmission 35 (1999), no. 3, 205-223. 
[21] T. Honold, Characterization of finite Frobenius rings. Arch. Math. (Basel) 76 no. 6, 406-415, 2001.

[22] S. L. Ma, A survey of partial difference sets. Designs, Codes and Cryptography, 4:221-261, 1994.

[23] A. A. Nechaev, Kerdock codes in a cyclic form, Discrete Math. Appl. 1 (1991), 365-384.

[24] G.-C. Rota. On the foundations of combinatorial theory I. Theory of Möbius functions. Zeitschrift für Wahrscheinlichkeitstheorie und Statistik, 2:340-368, 1964.

[25] R. P. Stanley, Enumerative combinatorics. Vol. 1, Cambridge University Press, Cambridge, 1997, With a foreword by Gian-Carlo Rota, Corrected reprint of the 1986 original.

[26] G. Törner and F. D. Veldkamp, Literature on geometry over rings. J. Geom. 42 (1991), no. $1-2,180-200$.

[27] Richard Wiegandt, On the general theory of Möbius inversion formula and Möbius product, Acta Sci. Math. Szeged 20 (1959), 164-180.

[28] J. A. Wood, Duality for modules over finite rings and applications to coding theory, Amer. J. Math. 121 (1999), no. 3, 555-575.

[29] J. A. Wood, Weight functions and the extension theorem for linear codes over finite rings, in Finite Fields: Theory, Applications and Algorithms, Contemp. Math. 225, Providence: Amer. Math. Soc., 1999, 231-243.

[30] J. A. Wood, Code equivalence characterizes finite Frobenius rings, preprint. To appear in Proc. Amer. Math. Soc. 2007. 